\title{
Surgical Management of Giant Walled-off Necrosis
}

\author{
Mehmet Akif Turkoglu*, Kursad Cifci, Veli Vural, Halil Erbis, Volkan Dogru and Ibrahim Aliosmanoglu \\ Department of General Surgery, Medicine School of Akdeniz University, Antalya, Turkey
}

\begin{abstract}
The term of Walled-off Pancreatic Necrosis (WOPN) is a new definition of the complicated Acute Necrotising Pancteatitis (ANP). WOPN is characterised with organised pancreatic necrosis tissue containing solid and fluid components. Conventionally, prophylactic antibiotic regimens are applied in some clinics, despite this argument is still controversary. We present here a case of huge WOPN treated with surgery and mentioned about our clinic management.
\end{abstract}

\section{Introduction}

In 2012 revision of the Atlanta Classificiation, WOPN was used to decribe the mature phase of Acute Necrotitising Pancteatitis (ANP), containing solid and liquid material surrounded by a mature, exaggerated wall made by reactive tissues [1]. Before WOPN, many terms were used to define this formation such as, organised pancreatic necrosis, necroma, pancreatic pseudocyst with necrosis, pancreatic sequestrum. WOPN can eventuate in \%1-9 of cases of ANPs. WOPN formation usually develops approximately 3-6 weeks after the ANP. Here in we present a case of 51 year old man who suffered from epigastric pain and early satiety related to WOPN.

\section{Presentation of Case}

A fifty one year-old male patient has been admitted to our clinic, with epigastric and back pain, early satiety. In preoperative findings there was no fever, elevation on leucocytes or pancreatic enzymes. On his medical history, he was hospitalised with severe acute pancreatitis 6 months ago and undergone cholecystectomy operation 2 years ago. During his follow-up period, multiphasic Computed Tomography (CT) images have shown a mass grown up to $16 \times 10 \mathrm{~cm}$, extending above the transverse mesocolon, reaching to sigmoid colon (Figure 1). The CT images revealed a hypodens mass compatible with WOPN, containing solid components and a walled-off image.

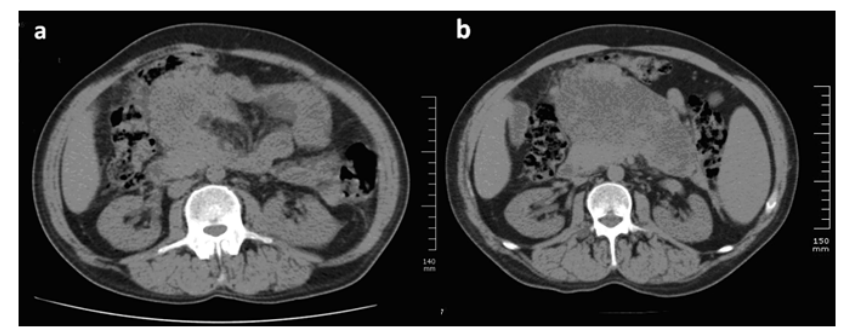

Figure 1: Axial computed tomography scan while acute severe pancreatitis attach (a) Axial Computed Tomography scan shows the WOPN.

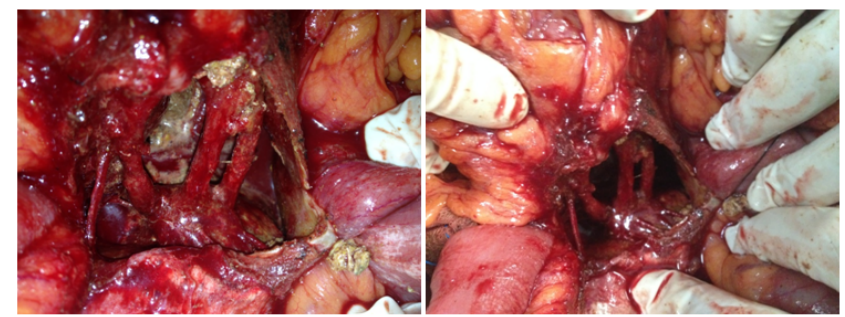

Figure 2: The branches of SMA and SMV lying inside WOPN cavity.
Firstly, diagnostic laparoscopy was apppointed for the initial surgical procedure. In laparoscopic exploration, it was seen that a big mass, adherent to duodenum and transverse colon, covered by omentum. According to laparoscopic exploration findings, laparotomy decision was made. After detaching the adhesions, and opening the wall of mass, it was seen that whole cavity filled by the slurry form of necrotising material. Superior Mesenteric Artery (SMA) and Superior Mesenteric Vein (SMV) branches were clearly observed that lying in the necrotising material (Figure 2). After debridement of necrosis, cavity was irrigated with hydrogene peroxyde aggresively, samples were taken for culture (Figure 3). Finally, two Jackson-Pratt drains are insterted into the cavity of WOPN and in the pouch of Douglas.

During the postoperative follow-up period at clinic, there has not been an elevation of leucocytes, C-Reactive Protein levels increased until $19 \mathrm{mg} / \mathrm{dl}$; started to regress at 3 days after operation. Although, imipenem therapy was started emprically immediately after surgery, he has no sign of infection, except postoperative second day's fever associated with atelectasis. However, peroperative cultures are confirmed with negative, thus prophylactic use of Imipenem was ceased at postoperative $4^{\text {th }}$ day. Pelvic drain was removed at postoperative

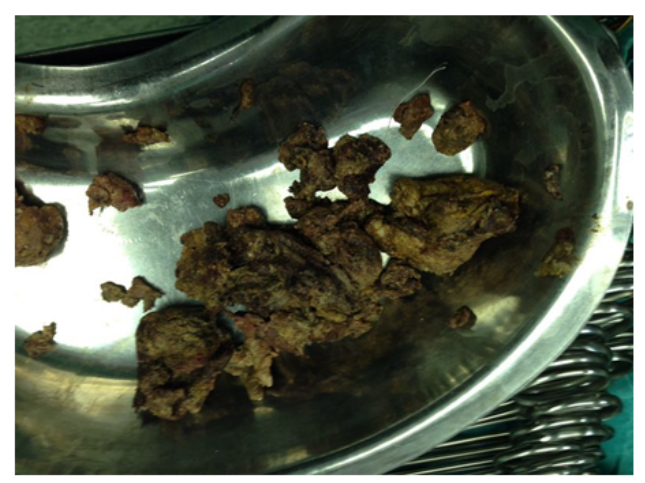

Figure 3: The solid content of WOPN cavity.

*Corresponding author: Mehmet Akif Turkoglu, Department of General Surgery, Medicine School of Akdeniz University, Antalya, Turkey, Tel: +905068864003; E-mail: makturko@gmail.com

Received March 05, 2014; Accepted April 20, 2014; Published April 29, 2014

Citation: Turkoglu MA, Cifci K, Vural V, Erbis H, Dogru V, et al. (2014) Surgical Management of Giant Walled-off Necrosis. Surgery Curr Res 4: 190 doi:10.4172/2161-1076.1000190

Copyright: (c) 2014 Turkoglu MA, et al. This is an open-access article distributed under the terms of the Creative Commons Attribution License, which permits unrestricted use, distribution, and reproduction in any medium, provided the original author and source are credited. 
$7^{\text {th }}$ day, the drainage of the WOPN cavity has fallen below $25 \mathrm{ml}$ at postoperative $14^{\text {th }}$ day and then removed. The patient was discharged smoothly 16 days after operation.

\section{Discussion}

In WOPN reactive tissues creates a sort of fibrous wall containing necrotic components. This necrotic pancreatic parenchyma and/or necrotic peripancreatic tissues is the site where WOPN derives and in time lesion could be infected, sometimes multiple, and may be present at sites distant from the pancreas [1-3]. Although WOPN is usually seen as aseptic necrosis, the most common seen bacterias in infected WOPN are E.Coli, K.Pneumoniae, E.Faecalis and S.Aureus [3,4]. In our case, the peroperative culture result was negative.

There is still no typical laboratory or radiological finding for determination of WOPN. In case of infection in WOPN, gas image can be shown in Computed Tomography (CT), but not always the exact finding of infection. Pancreatic enzyme levels usually are not correlated with the severity or quantity of necrosis. Spontaneous fistulisation to duodenum or colon can be seen on CT findings. For the differaintial diagnosis between pancreatic pseudocyst and WOPN, it has been shown the CT about $79-84 \%$ of cases [5]. The emphasis of differantial diagnosis between WOPN and pancreatic pseudocyst is critical because of determining of the choice of treatment.

There are several treatment options available for WOPN, such as percutaneous drainage, endoscopic drainage, laparoscopic drainage and surgical necrosectomy. Percutaneous drainage mostly fails, beacuse of the solid components of WOPN [6]. Also, in our case, percutaneous drainage has been tried before, but it was unsuccessful (Figure 3). Transmural endoscopic debridement is used treatment choice for most of the cases. Surgery is recommended for the cases, with giant WOPN formation over $15 \mathrm{~cm}$ or affecting other adjacent organs by adhering [2,7].

Prophylactic antibiotic use on a routine basis is not recommended when sterile necrosis present in severe ANP [8]. When percutaneous drainage from a necrotic site reveals an infection, pancreatic necrous penetrating antibiotics may be useful in saving time before intervention and they have demonstrated benefits in decreasing morbidity and mortality [8]. Nevertheless, to our best knowledge, at the postoperative period, there is still no consensus for the prophlactic use of antibiotics after surgical necrosectomy. Antibiotic habit of surgeons even when negative culture results reported still remains to be a trigger to our selfcriticism.

\section{Conclusion}

When severe acute necrotising pancreatitis and its complications occured, such as WOPN, percutaneous drainage and endoscopic interventions may fail. At this time, surgery should be considered as a solution. Unless the symptoms and clinical findings of infection are not determined clearly it is better to bear it in mind that the lesion can be aseptic. As in our case, the usage of prophylactic antibiotics can be unnessesary if the peroperative culture is negative. Nevertheless, it's hard to draw conclusions with relying on a single case, in the lack of clinical symptoms and laboratory findings of infection, omission of antibiotic use can be feasible during the management of walledoff necrosis at postoperative period. However the need to justify our actions and decisions, large series are required.

\section{References}

1. Banks PA, Bollen TL, Dervenis C, Gooszen HG, Johnson CD, et al. (2013) Classification of acute pancreatitis--2012: revision of the Atlanta classification and definitions by international consensus. Gut 62: 102-111.

2. Papachristou GI, Takahashi N, Chahal P, Sarr MG, Baron TH (2007) Peroral endoscopic drainage/debridement of walled-off pancreatic necrosis. Ann Surg 245: 943-951.

3. Munene G, Dixon E, Sutherland F (2011) Open transgastric debridement and internal drainage of symptomatic non-infected walled-off pancreatic necrosis. HPB (Oxford) 13: 234-239.

4. Stamatakos M, Stefanaki C, Kontzoglou K, Stergiopoulos S, Giannopoulos G, et al. (2010) Walled-off pancreatic necrosis. World J Gastroenterol 16: 17071712.

5. Takahashi N, Papachristou GI, Schmit GD, Chahal P, LeRoy AJ, et al. (2008) CT findings of walled-off pancreatic necrosis (WOPN): differentiation from pseudocyst and prediction of outcome after endoscopic therapy. Eur Radiol 18: 2522-2529.

6. Fischer A, Schrag HJ, Keck T, Hopt UT, Utzolino S (2008) Debridement and drainage of walled-off pancreatic necrosis by a novel laparoendoscopic rendezvous maneuver: experience with 6 cases. Gastrointest Endosc 67: 871878.

7. Gardner TB, Chahal P, Papachristou GI, Vege SS, Petersen BT, et al. (2009) A comparison of direct endoscopic necrosectomy with transmural endoscopic drainage for the treatment of walled-off pancreatic necrosis. Gastrointest Endosc 69: 1085-1094.

8. Tenner S, Baillie J, DeWitt J, Vege SS; American College of Gastroenterology (2013) American College of Gastroenterology guideline: management of acute pancreatitis. Am J Gastroenterol 108: 1400-1415. 